\title{
Dilatação por cateter-balão (dacrioplastia) para tratamento das obstruções congênitas do ducto lacrimonasal
}

\author{
Balloon-catheter dilatation (dacrioplasty) for \\ congenital nasolacrimal duct obstruction treatment
}

\author{
Roberto Murillo Limongi de Souza Carvalho ${ }^{1}$ \\ Luig'i Batista Seronni² \\ Marcos Ávila ${ }^{3}$
}

Trabalho realizado no Setor de Plástica Ocular do Centro de Referência em Oftalmologia (CEROF), da Universidade Federal de Goiás - UFG - Goiânia (GO) - Brasil.

${ }^{1}$ Chefe do Setor de Plástica Ocular do Centro de Referência em Oftalmologia (CEROF), da Faculdade de Medicina da Universidade Federal de Goiás - UFG - Goiânia (GO) - Brasil.

${ }^{2}$ Médicos Assistentes do Setor de Plástica Ocular do Centro de Referência em Oftalmologia (CEROF), da UFG - Goiânia (GO) - Brasil.

${ }^{3}$ Professor, Doutor e Chefe do Serviço de Oftalmologia da UFG - Goiânia (GO) - Brasil.

Endereço para correspondência: Roberto Murillo Limongi de Souza Carvalho. Rua T 47 Q 31 L3/5, Ed. Monte Carlo - Apto. 1.102 - Setor Bueno - Goiânia (GO) CEP 74210-180

E-mail: rmurillousp@hotmail.com

Recebido para publicação em 29.09.2007

Última versão recebida em 23.09.2009

Aprovação em 25.09.2009

\section{RESUMO}

Objetivo: Verificar o resultado da técnica, inédita no Brasil, de dilatação do ducto lacrimonasal usando cateter-balão para tratamento da obstrução congênita do ducto lacrimonasal. Métodos: Foram tratadas crianças com idade entre dois e cinco anos, com obstrução congênita de vias lacrimais usando o cateter-balão (Lacricath ${ }^{\circledR}$ ) para dilatação das vias lacrimais. O cateter foi introduzido pelo ponto lacrimal superior, sob anestesia geral. Resultados: Entre os 27 olhos tratados, 23 apresentaram desaparecimento dos sinais e sintomas de lacrimejamento (taxa de sucesso de $85,2 \%$ ). Todos os procedimentos foram realizados sem intercorrências e o seguimento pós-operatório mínimo foi de um ano. Conclusão: A dilatação com balão mostrou-se segura e eficiente como primeiro procedimento para os casos de obstrução congênita das vias lacrimais em crianças com mais de dois anos.

Descritores: Obstrução dos ductos lacrimais/congênito; Dilatação com balão/métodos; Crianças

\section{INTRODUÇÃO}

A obstrução congênita do ducto lacrimonasal (OCDLN) ocorre em aproximadamente $6 \%$ dos recém-nascidos, mais comumente por imperfuração da válvula de Hasner, em decorrência da falta de canalização do ducto na região de transição com a mucosa nasal, na altura do meato inferior ${ }^{(1)}$.

A dacrioestenose congênita pode ser tratada inicialmente de forma conservadora porque as obstruções podem se resolver espontaneamente no primeiro ano de $v_{i d a}{ }^{(2)}$. Massagem digital de Crigler e colírios antibióticos são os mais recomendados inicialmente ${ }^{(3-4)}$. Para crianças com lacrimejamento persistente, uma simples sondagem do ducto lacrimonasal pode ser terapêutica em $90 \%$ dos $\operatorname{casos}^{(5-6)}$. Quando a primeira sondagem não resolve a epífora, novas opções terapêuticas devem ser escolhidas, como a entubação das vias lacrimais com fio de silicone ${ }^{(7)}$. Complicações, incluindo deslocamento prematuro ou acidental do silicone e lesão do ponto lacrimal e córnea, têm sido relatados ${ }^{(8-10)}$.

A cateterização por balão, descrita por Becker e Berry ${ }^{(11)}$, tem sido usada com sucesso no mundo todo, sendo indicada para as crianças portadoras de OCDLN que já são mais velhas e aquelas que não tiveram sucesso com a sondagem. Um balão inflável de poliuretano na ponta de uma sonda guia semiflexível é inserida no ducto lacrimonasal e insuflada quando a mesma 
se encontra no nível da válvula de Hasner. Até o momento, não existem estudos relatando esta técnica na literatura oftalmológica brasileira.

O objetivo deste estudo é verificar o valor da dilatação das vias lacrimais baixas usando balão em portadoras de OCDLN, com idade acima de dois anos.

\section{MÉTODOS}

Este é um estudo prospectivo, intervencional, tipo série de casos.

O protocolo do estudo e Termo de Consentimento Livre e Esclarecido foram aprovados dos Conselhos de Ética Médica do Hospital das Clínicas da Universidade Federal de Goiás e pelo CONEP (Conselho Nacional de Ética e Pesquisa).

Dele participaram crianças com idade superior a dois anos e menores que cinco anos de idade, atendidos no ambulatório do Centro de Referência em Oftalmologia (CEROF), do Hospital das Clínicas da Universidade Federal de Goiás, com diagnóstico de obstrução congênita do ducto lacrimonasal. O diagnóstico de obstrução congênita foi feito por meio da presença dos sintomas e sinais clínicos de epífora, e confirmados pelo teste de desaparecimento da fluoresceína (Teste de "Milder").

Foram excluídos os pacientes que apresentaram: ectrópio de ponto lacrimal ou da pálpebra inferior, estenose importante de ponto lacrimal, lagoftalmo paralítico, alterações na estrutura palpebral que pudessem causar lacrimejamento (triquíase, entrópio), inflamações ou emergências oculares. Todos os pacientes incluídos neste estudo nunca haviam sido submetidos a nenhum procedimento cirúrgico anterior, tais como: sondagens, entubação com silicone ou dacriocistorrinostomia.

Procedimento: a cateterização foi realizada sob anestesia geral, de acordo com o protocolo padrão descrito por Becker e Berry $^{(11)}$. O cateter-balão (Lacricath ${ }^{\circledR}$ - Atrion Medical, Birmingham, Ala, U.S.A.) vazio foi introduzido pelo ponto lacrimal dilatado e posicionado na parte distal do ducto lacrimonasal. Isto foi possível por meio da verificação da presença da "marca" superior da ponta do cateter no ponto lacrimal (Figura 1). Após este tempo o balão foi inflado por 90 segundos com uma pressão em torno de $8 \mathrm{~atm}$. Em seguida, foi esvaziado e inflado

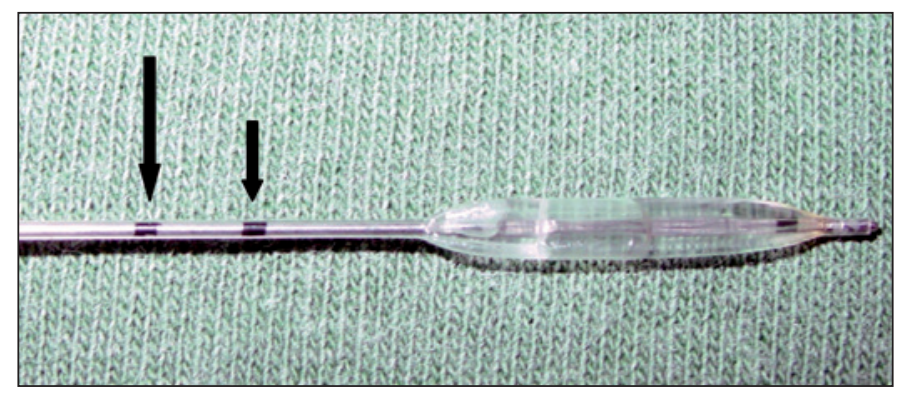

Figura 1 - Fotografia do Lacricath ${ }^{\circledR}$ (Atrion Medical, Birmingham, Ala, U.S.A). Observar a terminação deste cateter que é arredondada e não cortante, seguida da área que se insufla ("balão"). As marcas existentes na haste do cateter-balão servem como guia para a sua localização. novamente por mais 60 segundos. Este mesmo procedimento foi realizado novamente com a sonda posicionada em outro local, retirando $5 \mathrm{~mm}$ do cateter para fora do ponto lacrimal e posicionando neste a marca inferior do cateter (Figura 1). Após o procedimento foram prescritos colírios contendo associação de antibiótico e corticóide de 3/3 horas por 15 dias.

Os pacientes foram avaliados no Ambulatório de Vias lacrimais do Centro de Referência em Oftalmologia (CEROF), do Hospital das Clínicas da Universidade Federal de Goiás na terceira semana após o procedimento, no sexto mês e um ano depois. O sucesso do procedimento foi definido como desaparecimento dos sinais e sintomas de epífora e confirmado por meio do teste do desaparecimento da fluoresceína. O seguimento pós-operatório mínimo foi de um ano.

\section{RESULTADOS}

Vinte e sete vias lacrimais de 21 crianças com OCDLN foram tratadas com a dilatação por cateter-balão. Entre estas, 6 apresentavam obstrução bilateral e 15 unilateral. A idade variou entre dois e cinco anos, com média de idade de 38 meses. Entre as 27 vias lacrimais tratadas, 23 apresentaram desaparecimento dos sinais e sintomas de lacrimejamento (taxa de sucesso de $85,2 \%$ ).

O quadro 1 mostra a distribuição das crianças por faixa etária. Na faixa etária entre 24 e 36 meses houve sucesso de $100 \%$. $\mathrm{Na}$ faixa etária entre 37 e 48 meses a taxa de sucesso caiu para $87,5 \%$ e de $57,1 \%$ para as crianças entre 49 e 60 meses.

A tabela 1 revela as principais características dos casos que não apresentaram melhora. Entre os 4 casos de insucesso, 2 apresentaram melhora importante dos sinais e sintomas de epífora até os primeiros dois meses após o procedimento, porém com piora dos mesmos nos meses subsequentes.

Nenhuma criança apresentou complicações durante ou após o procedimento, tais como edema periorbitário, sangramento, celulite ou dor.

\section{DISCUSS ÃO}

Cerca de $20 \%$ de todos os recém-nascidos apresentam disfunção do sistema de drenagem lacrimal e apresentarão lacrimejamento no primeiro ano de vida. Entre todas as doenças passíveis de tratamento na oftalmologia pediátrica, a OCDLN é uma das mais controversas.

Apesar da sondagem lacrimal possuir taxa de sucesso de aproximadamente $90 \%$, a maioria dos estudos mostram um decréscimo na taxa de sucesso com o aumento da idade do paciente ${ }^{(12-15)}$. Assim, em alguns casos há que se utilizar outras opções terapêuticas.

A cateterização por balão (dacrioplastia), usada após falha com a sondagem, tem tido taxas de sucesso variando entre $74 \%$ e $94 \%{ }^{(16)}$. Recentemente foi relatada taxa de cura de $82,1 \%$ com o uso da dilatação por balão para os pacientes que não obtiveram sucesso com a sondagem ${ }^{(17)}$. 


\begin{tabular}{|c|c|c|c|}
\hline \multirow[t]{2}{*}{ Faixa etária } & \multicolumn{3}{|c|}{ Melhora com sondagem } \\
\hline & Sim & Não & Total \\
\hline 24 meses até 36 meses & $12(100 \%)$ & $0(0 \%)$ & 12 \\
\hline 36 meses até 48 meses & $7(87,5 \%)$ & $1(12,5 \%)$ & 8 \\
\hline 48 meses até 60 meses & $4(57,1 \%)$ & $3(42,9 \%)$ & 7 \\
\hline
\end{tabular}

\begin{tabular}{|c|c|c|c|c|c|}
\hline Casos & Idade & Sexo & $\begin{array}{c}\text { Presença de } \\
\text { secreção ocular }\end{array}$ & $\begin{array}{l}\text { Expressão do } \\
\text { saco lacrimal }\end{array}$ & $\begin{array}{c}\text { Dacriocistite } \\
\text { prévia }\end{array}$ \\
\hline 1 & 3 anos e 3 meses & Masculino & Sim & Positiva & Não \\
\hline 2 & 4 anos & Feminino & Sim & Positiva & Não \\
\hline 3 & 5 anos & Masculino & Não & Negativa & Não \\
\hline 4 & 5 anos & Masculino & Não & Negativa & Não \\
\hline
\end{tabular}

O nosso estudo, apesar de ter sido realizado com crianças mais velhas, com média de idade de 38 meses, mostrou que a dacrioplastia usada como primeiro procedimento teve taxa de sucesso semelhante, alcançando $85,2 \%$, o que também está de acordo com os estudos realizados em outros países, onde a taxa de sucesso variou entre $79 \%$ e $96 \%$ quando a dilatação foi usada como primeiro procedimento, em crianças acima de um ano de idade ${ }^{(18-20)}$.

Entre os 4 casos de insucesso com a dilatação pelo cateterbalão, 3 apresentaram idade entre 49 e 60 meses, mostrando um decréscimo importante do sucesso após os quatro anos de idade $(57,1 \%)$. Ainda, entre os casos de insucesso, esperaríamos que a presença de secreção ocular e expressão positiva do saco lacrimal fossem encontrados nas crianças mais velhas, visto que a dilatação a jusante das vias lacrimais obstruídas seria mais provável. Entretanto isto não foi constatado (Tabela 1).

É importante relatar que entre os 4 casos de insucesso, 2 apresentaram uma melhora importante dos sinais e sintomas de epífora até os primeiros dois meses após o procedimento, porém com piora nos meses subsequentes. As hipóteses para isso seria a reestenose do ducto lacrimonasal, o que nos levou a submeter estas crianças a dacriocistorrinostomia.

A limitação do presente estudo é sua natureza não-mascarada. Um estudo prospectivo randomizado mascarado seria necessário para comprovar a real efetividade da dacrioplastia. Como as taxas de sucesso com as outras modalidades de tratamento (sondagens repetidas e entubação com silicone) também são relativamente altas, este tipo de estudo iria requerer um grande número de pacientes o que tornaria um estudo de difícil execução.

Entretanto, por se tratar de doença de alta prevalência em crianças que procuraram o Sistema Único de Saúde (SUS), a metodologia aqui avaliada se mostrou segura e eficiente com a vantagem de baixo custo, simples execução e de fácil acom- panhamento após o procedimento, razões que a tornaram o método de escolha em nosso meio para crianças com mais de dois anos de idade. O intuito é de se evitar a cirurgia de entubação de vias lacrimais e a dacriocistorrinostomia, condutas que são mais agressivas, de execução mais difícil e com pós-operatório mais complicado.

Assim, os autores acreditam que esforços devam ser iniciados para sua padronização pelo SUS.

\section{CONCLUS ÃO}

A dilatação com balão mostrou-se segura e eficiente como primeiro procedimento para crianças portadoras de obstrução congênita das vias lacrimais com idade acima de dois anos.

\section{ABSTRACT}

Purpose: To verify the results of balloon-catheter dilatation for the treatment of congenital lacrimal duct obstruction. To the best of author's knowledge this procedure has not been previously used in Brazil and deserves scientific studies. Methods: Children between 2 and 5 years old, with congenital lacrimal duct obstruction, were treated with balloon-catheter dilatation $\left(\right.$ Lacricath $\left.^{\circledR}\right)$. Catheter was introduced by the superior lacrimal punctum, under general anesthesia. Results: Twenty-seven eyes were treated and 23 presented total improvement of epiphora signs and symptoms ( $85.2 \%$ success rate). During a one year follow-up, all the procedures had good outcomes, without complications and all the children had 1 year of follow-up. Conclusion: Balloon-catheter dilatation is an efficient and safe procedure and might be used for congenital lacrimal duct obstruction treatment in children older than 2 years. 
Keywords: Lacrimal duct obstruction/congenital; Balloon dilatation/methods; Children

\section{REFERÊNCIAS}

1. Paul TO. Medical management of congenital nasolacrimal duct obstruction. J Pediatr Ophthalmol Strabismus. 1985;22(2):68-70.

2. Katowitz JA, Welsh MG. Timing of initial probing and irrigation in congenital nasolacrimal duct obstruction. Ophthalmology. 1987;94(6):698-705.

3. Crigler LW. The treatment of congenital dacryocystitis. J Am Med Assoc. 1923;81(1):23-4

4. Castelo Branco Neto E, Castelo Branco B, Cardoso CC, Carvalho RG, Mota E, Castelo Branco A. Tratamento das obstruções congênitas do ducto nasolacrimal. Arq Bras Oftalmol. 2009;72(1):75-8.

5. American Academy of Ophthalmology. Basic Clinical Science Course, Section 6: Pediatric Ophthalmology and Strabismus. San Francisco: American Academy of Ophthalmology; 2002. p.492.

6. O'Donell BA, Adenis JP, Linberg JV, Rose GE, Sullivan TJ, Wobig JL. The failed probing. Clin Experiment Ophthalmol. 2001;29(5):276-80.

7. Migliori ME, Putterman AM. Silicone intubation for the treatment of congenital lacrimal duct obstruction: successful results removing the tubes after six weeks. Ophthalmology. 1988;95(6):792-5.

8. Dortzbach RK, France TD, Kushner BJ, Gonnering RS. Silicone intubation for obstruction of the nasolacrimal duct in children. Am J Ophthalmol. 1982; 94(5):585-90.

9. Leone CR Jr, Van Gemert JV. The success rate of silicone intubation in congenital lacrimal obstruction. Ophthalmic Surg. 1990;21(2):90-2.
10. Ratliff CD, Meyer DR. Silicone intubation without intranasal fixation for treatment of congenital nasolacrimal duct obstruction. Am J Ophthalmol. 1994;118(6):781-5.

11. Becker BB, Berry FD. Balloon catheter dilatation in pediatric patients. Ophthalmic Surg. 1991;22(12):750-2.

12. Schellini SA, Narikawa S, Ribeiro SCF, Nakagima V, Padovani CR, Padovani CRP. Obstrução nasolacrimal congênita: fatores relacionados com a melhora após sondagem terapêutica. Arq Bras Oftalmol. 2005;68(5):627-30.

13. Mannor GE, Rose GE, Frimpong-Ansah K, Ezra E. Factors affecting the success of nasolacrimal duct probing for congenital nasolacrimal duct obstruction. Am J Ophthalmol. 1999;127(5):616-7.

14. Robb RM. Success rates of nasolacrimal duct probing at time intervals after 1 year of age. Ophthalmology. 1998;105(7):1307-9; discussion 1309-10.

15. Kushner BJ. The management of nasolacrimal duct obstruction in children between 18 months and 4 years old. J AAPOS. 1998;2(1):57-60.

16. Becker BB, Berry FD, Koller H. Balloon catheter dilatation for treatment of congenital nasolacrimal duct obstruction. Am J Ophthalmol. 1996;121(3):304-9.

17. Casady DR, Meyer DR, Simon JW, Stasior GO, Zobal-Ratner JL. Stepwise treatment paradigm for congenital nasolacrimal duct obstruction. Ophthal Plast Reconstr Surg. 2006;22(4):243-7.

18. Tao S, Meyer DR, Simon JW, Zobal-Ratner J. Success of balloon catheter dilatation as a primary or secondary procedure for congenital nasolacrimal duct obstruction. Ophthalmology. 2002;109(11):2108-11. Comment in: Ophthalmology. 2003;110(10):2069-70; author reply 2070-1.

19. Lueder GT. Balloon catheter dilation for treatment of persistent nasolacrimal duct obstruction. Am J Ophthalmol. 2002;133(3):337-40.

20. Lueder GT. Balloon catheter dilation for treatment of older children with nasolacrimal duct obstruction. Arch Ophthalmol. 2002;120(12):1685-8. Comment in: Arch Ophthalmol. 2003;121(12):1804; author reply 1804-5. 